\title{
Semiconductor UV photonics: feature introduction
}

\author{
Xiaohang Li, ${ }^{1, *}$ (1) Russell D. Dupuis, ${ }^{2}$ and Tim Wernicke ${ }^{3}$ \\ ${ }^{1}$ King Abdullah University of Science and Technology (KAUST), Advanced Semiconductor Laboratory, Thuwal 23955-6900, Saudi Arabia \\ ${ }^{2}$ School of Electrical and Computer Engineering, Georgia Institute of Technology, Atlanta, Georgia 30332, USA \\ ${ }^{3}$ Technische Universität Berlin, Institute of Solid State Physics, Berlin D-10623, Germany \\ *Corresponding author: xiaohang.li@kaust.edu.sa
}

Received 18 November 2019; posted 18 November 2019 (Doc. ID 383804); published 1 December 2019

\begin{abstract}
Semiconductor UV photonics research has emerged as one of the most heavily invested areas among semiconductor photonics research due to numerous crucial applications such as sterilization, sensing, curing, and communication. The feature issue disseminates nine timely original research and two review papers from leading research groups and companies, covering most frontiers of the semiconductor UV photonics research, from epitaxy, device physics and design, nanostructures, fabrication, packaging, reliability, and application for light-emitting diodes, laser diodes, and photodetectors. () 2019 Chinese Laser Press
\end{abstract}

https://doi.org/10.1364/PRJ.7.0SUVP1

The year of 2019 marks the 30th anniversary of the invention of the first GaN PN junction light-emitting diode (LED), which was later recognized by the 2014 Nobel Prize in Physics [1]. This very first GaN LED was actually a UVA LED operating at $\sim 375 \mathrm{~nm}$. In the decades since 1989 , however, the research community has focused on indium-incorporated visible InGaN LED for solid-state lighting and display and has made tremendous progress. Today, the peak external quantum efficiency (EQE) of most blue LEDs in production can reach over $80 \%$ and that of yellow LEDs has exceeded 30\% [2] .

In comparison, the progress at the shorter-wavelength end of the spectrum has been more moderate for UV devices. The UV devices typically comprise larger-bandgap materials such as $\mathrm{Al}$-rich AlGaN. Larger bandgap increases the activation energy of the typical p-and n-type dopants, thus causing lower layer conductivity. Moreover, the conduction band minimum and the valence band maximum of the larger-bandgap materials further drift away from the workfunction of common metals for contact, leading to a larger Schottky barrier and hence increased contact resistivity. The use of the larger-bandgap materials can also hinder out-of-plane carrier transport due to reduced carrier mobility, compromising injection efficiency. Additionally, the UV emitters can suffer low light extraction efficiency due to absorption of the p-type region and enhanced transversemagnetic (TM) portion of the emission amid the rising split-off (SO) band. Consequently, most of the UV devices still suffer low performance. Specifically, technically-important commercial UVB and UVC LEDs still operate at a low $\mathrm{EQE}$ of a few percent [3], which is much lower than that of competing technologies including mercury lamps. The development of UV lasers has been even slower, where the first electrically-injected UVC laser diode (LD) has just been demonstrated and the wavelength is limited at $271.8 \mathrm{~nm}$ [4].
Therefore, there still remains significant challenges and opportunities ahead for semiconductor UV photonics research.

The feature issue includes 11 original research and review papers tackling most major aspects of semiconductor UV photonics research including LEDs, LDs, and photodetectors (PDs): epitaxy, characterization, processing, device physics, nanostructures, device reliability, and UV-based communication. They expose cutting-edge research of semiconductor UV photonics and can benefit a broad range of readership.

Kuhn et al. have demonstrated tunnel junction UVC LEDs by metalorganic vapor phase epitaxy (MOVPE) for the first time to eliminate the absorbing p-type layers [5], while most previous attempts have relied on molecular beam epitaxy (MBE) to do so. For characterization of UV materials and structures, Trager-Cowan et al. have shown that nondestructive SEM techniques can provide in-depth insights on topography, defects, composition, doping, and light emission [6]. Apart from epitaxy, the processing of UV devices is equally critical to tap into devices with greater performance, compatibility, and architecture. $\mathrm{Xu}$ et al. have demonstrated high-performance GaN-based photodiodes via the CMOScompatible Mg implantation rather than MOVPE in-situ doping and have developed the relevant physics model [7]. Wang et al. have performed fabrication of microdisks with air-bridge electrodes and realized the first electrically-injected UV lasing at $386.3 \mathrm{~nm}$ based on the microdisk structure [8]. Nagasawa and Hirano from UV Craftory provide a rare but highly-valuable review of encapsulation materials and packaging for UVC LEDs, which are closely related to light extraction efficiency, the major EQE limiting factor [9]. He et al. have applied the micro-LED fabrication technique to enable a record data transmission rate over 1 Gbps for UVC communication, which is one of the most important applications of UV devices [10]. 
Due to much smaller mobility than electrons and a potential barrier at the p-type electron blocking layer (EBL), holes have a significantly lower injection efficiency. Zhang et al. have introduced a composition-graded EBL to mitigate the polarizationinduced electric field at the EBL to enhance hole injection. The corresponding experiment achieved a high EQE of $7.6 \%$ for 275 nm UVC LEDs [11]. Nanorods and nanostructures are important ways of enhancing device efficiency due to advantageous structural and electronic properties. Liu et al. have systematically illustrated the design and growth of nanocrystals by molecular beam epitaxy (MBE) to enhance performance of UV LEDs and lasers [12]. Armstrong et al. have employed nanodotbased optically active floating gate for $\mathrm{AlGaN}$-channel high electron mobility transistor (HEMT) PDs to demonstrate large responsivity, moderate bandwidth, and low DC power loss [13]. Zhang et al. have fabricated nanorod UVC LEDs at $274 \mathrm{~nm}$ by utilizing nanosphere lithography and dry-etching technique on MOVPE-grown wafers. The nanorod UVC LEDs show 2.5 times EQE enhancement thanks to transformation of carriers from excitons to free electron-hole pairs and higher light extraction efficiency [14]. Since the UV device $\mathrm{EQE}$ is still low in general, the majority of the input energy would not convert to light. This poses greater challenges to device reliability and lifetime. Ruschel et al. have performed a timely study on the degradation of UVB LEDs. They show that higher current can strongly accelerate device aging, which also involves Auger recombination. Thus, lower operation current and adjustment of carrier distribution would improve device lifetime significantly [15].

\section{REFERENCES}

1. H. Amano, M. Kito, K. Hiramatsu, and I. Akasaki, "P-type conduction in Mg-doped GaN treated with low-energy electron beam irradiation (LEEBI)," Japanese J. Appl. Phys. 28, L2112-L2114 (1989).

2. F. Jiang, J. Zhang, L. Xu, J. Ding, G. Wang, X. Wu, X. Wang, C. Mo, Z. Quan, X. Guo, C. Zheng, S. Pan, and J. Liu, "Efficient InGaN-based yellow-light-emitting diodes," Photon. Res. 7, 144-148 (2019).

3. M. Kneissl, T.-Y. Seong, J. Han, and H. Amano, "The emergence and prospects of deep-ultraviolet light-emitting diode technologies," Nat. Photonics 13, 233-244 (2019).

4. Z. Zhang, M. Kushimoto, T. Sakai, N. Sugiyama, L. J. Schowalter, C. Sasaoka, and H. Amano, "A $271.8 \mathrm{~nm}$ deep-ultraviolet laser diode for room temperature operation," Appl. Phys. Express 12, 124003 (2019).
5. C. Kuhn, L. Sulmoni, M. Guttmann, J. Glaab, N. Susilo, T. Wernicke, M. Weyers, and M. Kneissl, "MOVPE-grown AIGaN-based tunnel heterojunctions enabling fully transparent UVC LEDs," Photon. Res. 7, B7-B11 (2019).

6. C. Trager-Cowan, A. Alasmari, W. Avis, J. Bruckbauer, P. R. Edwards, B. Hourahine, S. Kraeusel, G. Kusch, R. Johnston, G. Naresh-Kumar, R. W. Martin, M. Nouf-Allehiani, E. Pascal, L. Spasevski, D. Thomson, S. Vespucci, P. J. Parbrook, M. D. Smith, J. Enslin, F. Mehnke, M. Kneissl, C. Kuhn, T. Wernicke, S. Hagedorn, A. Knauer, V. Kueller, S. Walde, M. Weyers, P.-M. Coulon, P. A. Shields, Y. Zhang, L. Jiu, Y. Gong, R. M. Smith, T. Wang, and A. Winkelmann, "Scanning electron microscopy as a flexible technique for investigating the properties of UV-emitting nitride semiconductor thin films," Photon. Res. 7, B73-B82 (2019).

7. W. Xu, Y. Shi, F. Ren, D. Zhou, L. Su, Q. Liu, L. Cheng, J. Ye, D. Chen, R. Zhang, Y. Zheng, and H. Lu, "Magnesium ion-implantation-based gallium nitride $p$-i-n photodiode for visible-blind ultraviolet detection," Photon. Res. 7, B48-B54 (2019).

8. J. Wang, M. Feng, R. Zhou, Q. Sun, J. Liu, Y. Huang, Y. Zhou, H. Gao, $X$. Zheng, M. Ikeda, and H. Yang, "GaN-based ultraviolet microdisk laser diode grown on Si," Photon. Res. 7, B32-B35 (2019).

9. Y. Nagasawa and A. Hirano, "Review of encapsulation materials for AlGaN-based deep-ultraviolet light-emitting diodes," Photon. Res. 7, B55-B65 (2019).

10. X. He, E. Xie, M. S. Islim, A. A. Purwita, J. J. D. McKendry, E. Gu, H. Haas, and M. D. Dawson, "1 Gbps free-space deep-ultraviolet communications based on III-nitride micro-LEDs emitting at $262 \mathrm{~nm}$," Photon. Res. 7, B41-B47 (2019).

11. Z.-H. Zhang, J. Kou, S.-W. H. Chen, H. Shao, J. Che, C. Chu, K. Tian, Y. Zhang, W. Bi, and H.-C. Kuo, "Increasing the hole energy by grading the alloy composition of the p-type electron blocking layer for very high-performance deep ultraviolet light-emitting diodes," Photon. Res. 7, B1-B6 (2019).

12. X. Liu, K. Mashooq, D. A. Laleyan, E. T. Reid, and Z. Mi, "AIGaN nanocrystals: building blocks for efficient ultraviolet optoelectronics," Photon. Res. 7, B12-B23 (2019).

13. A. M. Armstrong, B. A. Klein, A. A. Allerman, A. G. Baca, M. H. Crawford, J. Podkaminer, C. R. Perez, M. P. Siegal, E. A. Douglas, V. M. Abate, and F. Leonard, "Visible- and solar-blind photodetectors using AIGaN high electron mobility transistors with a nanodot-based floating gate," Photon. Res. 7, B24-B31 (2019).

14. L. Zhang, Y. Guo, J. Yan, Q. Wu, Y. Lu, Z. Wu, W. Gu, X. Wei, J. Wang, and $\mathrm{J}$. $\mathrm{Li}$, "Deep ultraviolet light-emitting diodes based on a well-ordered AlGaN nanorod array," Photon. Res. 7, B66-B72 (2019).

15. J. Ruschel, J. Glaab, B. Beidoun, N. L. Ploch, J. Rass, T. Kolbe, A. Knauer, M. Weyers, S. Einfeldt, and M. Kneissl, "Current-induced degradation and lifetime prediction of $310 \mathrm{~nm}$ ultraviolet light-emitting diodes," Photon. Res. 7, B36-B40 (2019). 